\title{
POST PANCREATITIS SMA SYNDROME: A CASE REPORT
}

\author{
Ritesh M. Bodade ${ }^{1}$, Asmita S. Dhurve ${ }^{2}$
}

\section{HOW TO CITE THIS ARTICLE:}

Ritesh M. Bodade, Asmita S. Dhurve. "Post Pancreatitis SMA Syndrome: A Case Report". Journal of Evolution of Medical and Dental Sciences 2015; Vol. 4, Issue 43, May 28; Page: 7552-7555,

DOI: $10.14260 /$ jemds/2015/1095

ABSTRACT: Superior mesenteric artery (SMA) syndrome is a rare acquired disorder in which acute angulation of SMA causes compression of the third part of the duodenum between the SMA and the aorta, leading to obstruction. Loss of fatty tissue as a result of a variety of debilitating conditions is believed to be the etiologic factor causing the acute angulation. Conditions like increased spinal lordosis, application of a body cast, short ligament of Treitz or unusually low origin of SMA may also precipitate this syndrome. The diagnosis of SMA syndrome is based on clinical symptoms and radiologic evidence of obstruction. SMA syndrome has been described after rapid or severe weight loss in conditions such as cancer or extensive burn injuries, prolonged bed rest, anorexia, or malabsorption syndromes. Herewith we are reporting a case of post pancreatitis SMA syndrome.

KEYWORDS: Post pancreatitis SMA syndrome; superior mesenteric artery syndrome; SMA syndrome.

CASE REPORT: A 20 year old thin built male presented with history of pain in upper abdomen and bilious vomiting since two days with past history of hospitalisations twice in previous month for acute pancreatitis. Patient was dehydrated and in compensated shock with fullness in upper abdomen and positive succussion splash. He was resuscitated and ryles tube aspiration done and his pain subsided immediately. Patient was managed conservatively. Ryles tube was removed on $3^{\text {rd }}$ day but he again developed pain and bilious vomiting. His haematological investigations, serum amylase and lipase, serum electrolytes were normal. X-ray abdomen [Fig. 1] showed dilatation of stomach and barium meal [Fig. 2] study showed dilatation of the first and second part of the duodenum, extrinsic compression of the third part and a collapsed distal small bowel. CECT abdomen [Fig. 3] showed dilatation of duodenum upto $3^{\text {rd }}$ part with compression of $3^{\text {rd }}$ part of duodenum between superior mesenteric artery anteriorly and aorta posteriorly. Aortomesenteric distance was reduced to $4 \mathrm{~mm}$, thus confirming SMA syndrome. Patient was treated conservatively with total parenteral nutrition for 25 days but without symptomatic benefit, so decision to do duodenojejunostomy (Fig. 4) was taken. Postoperatively patients symptoms improved dramatically he gained weight and vomiting subsided completely.

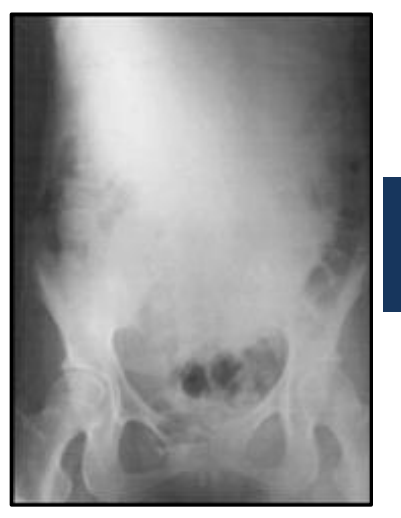

Fig. 1: Plain X-ray Abdomen showing Dilated stomach 


\section{CASE REPORT}

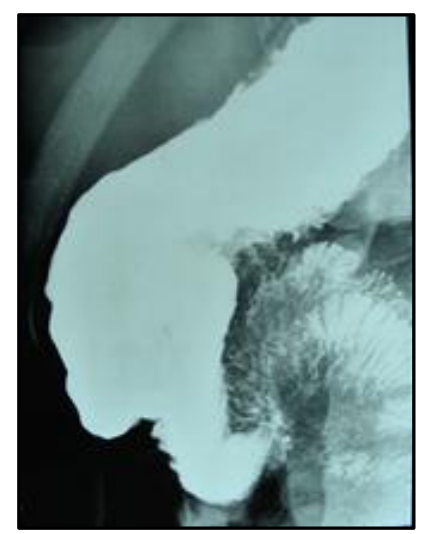

Fig. 2: Barium meal study showing dilatation of the first and second part of the duodenum

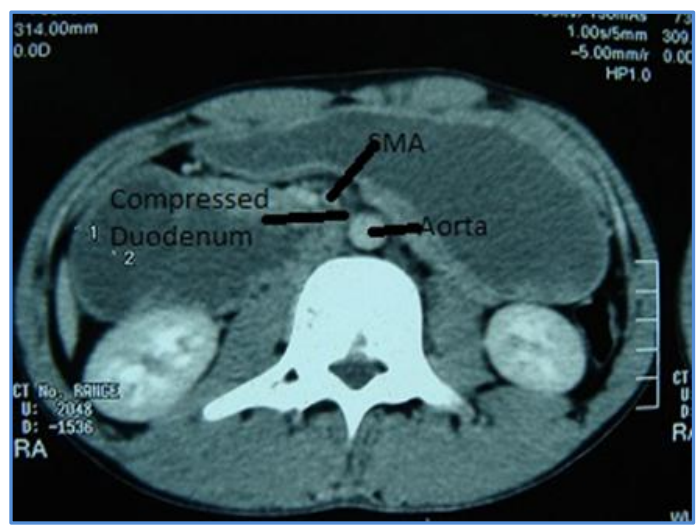

Fig. 3: CECT abdomen showed dilatation of duodenum upto $3^{\text {rd }}$ part with compression of $3^{\text {rd }}$ part of duodenum between superior mesenteric artery anteriorly and aorta posteriorly

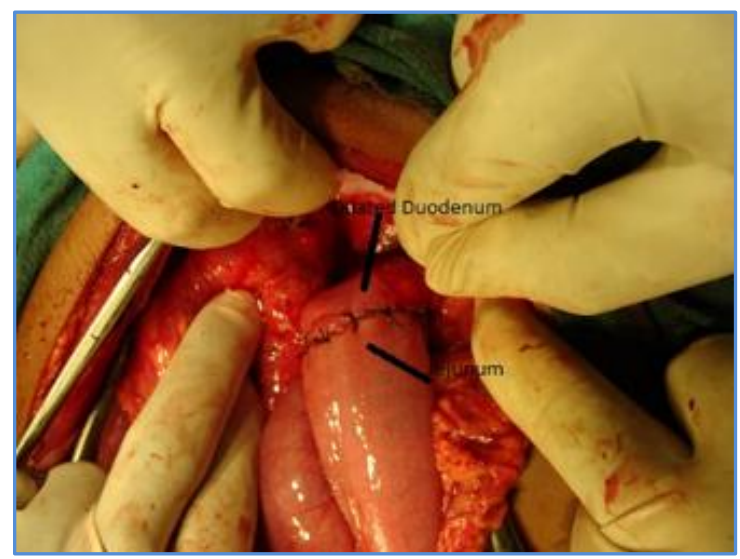

Fig. 4: Duodeno-jejunostomy 
DISCUSSION: SMA syndrome was first described by von Rokitanski in 1861. Later, Wilkie provided a more detailed clinical and pathophysiologic description in a series of 64 patients and suggested treatment approaches.[1] The defining feature is upper gastrointestinal obstruction caused by compression of the $3^{\text {rd }}$ part of the duodenum between the SMA anteriorly and the aorta posteriorly. Under conditions of severe weight loss, fat and lymphatic cushion around the SMA gets diminished, causing angulation and reduction in the distance between the aorta and the superior mesenteric artery. Normally, the aortomesenteric angle and aortomesenteric distance is $25^{\circ}$ to $60^{\circ}$ and 10 to 28 $\mathrm{mm}$, respectively. In SMA syndrome, both parameters are reduced, with values of $6^{\circ}$ to $15^{\circ}$ and 2 to 8 $\mathrm{mm} .{ }^{[2]}$ Increased spinal lordosis, application of a body cast, short ligament of Treitz, or unusually low origin of SMA may also precipitate this syndrome. The diagnosis of SMA syndrome is based on clinical symptoms and radiologic evidence of obstruction. Patients may present acutely, or chronic insidiously, or with an acute exacerbation of chronic symptoms. The acute presentation is characterized by signs and symptoms of duodenal obstruction. Chronic cases may present with longstanding vague abdominal symptoms, early satiety and anorexia, or recurrent episodes of abdominal pain and vomiting.

Plain radiograph demonstrates dilated, fluid- and gas-filled stomach. Barium radiography shows dilatation of the first and second part of the duodenum, extrinsic compression of the third part, and a collapsed distal small bowel. CECT scan or magnetic resonance angiography (MRA) enables visualization of vascular compression of the duodenum and measurement of aortomesenteric distance precisely. Both these procedures are non-invasive and are probably equivalent to angiography, which was previously considered reference standard for diagnosing SMA syndrome. ${ }^{[3,4]}$

Traditionally, treatment has been conservative with nasogastric decompression and hyperalimentation followed by oral feeding with frequent small meals. Surgery may be considered if conservative treatment fails. Duodenojejunostomy is effective in the majority of patients.[5] A 7-year follow-up study of 16 patients treated with duodenojejunostomy found that outcome was regarded as excellent by 3 patients, good by 6, satisfactory by 5, and poor by 2 patients.[6] Laparoscopic duodenojejunostomy offers a minimally invasive therapeutic approach to SMA syndrome.[7] Laparoscopic surgery involving lysis of the ligament of Treitz with mobilization of the duodenum.

Our patient had rapid weight loss in a month's duration secondary to two attacks of acute pancreatitis which may have caused SMA syndrome.

CONCLUSION: SMA syndrome should be always kept in mind while dealing with cases of proximal small bowel obstruction in patients suffering from diseases causing sudden weight loss. Aggressive diagnosis using upper GI barium studies and CT scan with individualised treatment has got good outcome.

\section{REFERENCES:}

1. Wilkie, D. Chronic duodenal ileus. Br J Surg. 1921: 204-14.

2. Lippl, F; Hannig, C; Weiss, W; Allescher, HD; Classen, M; Kurjak, M. Superior mesenteric artery syndrome: diagnosis and treatment from the gastroenterologist's view. J Gastroenterol. 2002; 37: 640-3.

3. Bedoya, R; Lagman, SM; Pennington, GP; Kirdnual, A. Clinical and radiological aspects of the superior mesenteric artery syndrome. J Fla Med Assoc. 1986; 73: 686-9. 


\section{CASE REPORT}

4. Gustafsson, L; Falk, A; Lukes, PJ; Gamklou, R. Diagnosis and treatment of superior mesenteric artery syndrome. Br J Surg. 1984; 71: 499-501.

5. Hines, JR; Gore, RM; Ballantyne, GH. Superior mesenteric artery syndrome. Diagnostic criteria and therapeutic approaches. Am J Surg. 1984; 148: 630-2.

6. Ylinen, P; Kinnunen, J; Hockerstedt, K. Superior mesenteric artery syndrome. A follow-up study of 16 operated patients. J Clin Gastroenterol. 1989; 11: 386-91.

7. Richardson, WS; Surowiec, WJ. Laparoscopic repair of superior mesenteric artery syndrome. Am J Surg. 2001; 181: 377-8.

\section{AUTHORS:}

1. Ritesh M. Bodade

2. Asmita S. Dhurve

\section{PARTICULARS OF CONTRIBUTORS:}

1. Assistant Professor, Department of General Surgery, IGGMC, Nagpur.

2. Assistant Professor, Department of General Surgery, GMC, Nagpur.

FINANCIAL OR OTHER COMPETING INTERESTS: None

\section{NAME ADDRESS EMAIL ID OF THE} CORRESPONDING AUTHOR:

Dr. Ritesh Bodade, Department of Surgery, IGGMC, CA Road, Nagpur, Maharashtra, India.

E-mail: dr.ritesh.bodade@gmail.com

Date of Submission: 16/05/2015. Date of Peer Review: 18/05/2015. Date of Acceptance: 21/05/2015. Date of Publishing: 28/05/2015. 